\title{
Controle de Dez espécies Daninhas em Cana-de-Açúcar com o Herbicida Mesotrione em Mistura com Ametryn e Metribuzin ${ }^{1}$
}

\author{
Control of Ten Weed Species in Sugarcane Using Mesotrione Mixed with Ametryn and Metribuzin
}

CARVALHO, F.T. ${ }^{2}$, CASTRO, R.M..$^{3}$, OTSUBO, R.I. ${ }^{3}$ e PEREIRA, F.A.R. ${ }^{4}$

\begin{abstract}
RESUMO - Existem muitas opções de herbicidas para aplicações em pré-emergência em cana-de-açúcar, mas o grande desafio atual para a cultura é o controle pós-emergente. O objetivo deste trabalho foi avaliar a eficácia do herbicida mesotrione em mistura com ametryn e metribuzin no controle em pós-emergência de 10 espécies daninhas semeadas em canade-açúcar, variedade RB 86-7515. As espécies daninhas selecionadas para o experimento foram: Brachiaria decumbens, Brachiaria plantaginea, Cenchrus echinatus, Digitaria horizontalis, Panicum maximum, Amaranthus deflexus, Bidens pilosa, Euphorbia heterophylla, Ipomoea nil e Sida glaziovii, semeadas nas entrelinhas após a emergência da cultura. Os herbicidas foram aplicados aos 45 dias após o plantio da cana-de-açúcar, com as plantas daninhas monocotiledôneas na fase de terceiro perfilho e as dicotiledôneas com três a quatro pares de folhas, e constaram dos seguintes tratamentos: mesotrione (120 $\left.\mathrm{g} \mathrm{ha}^{-1}\right)$; ametryn (2.000 $\left.\mathrm{g} \mathrm{ha}^{-1}\right)$; metribuzin $\left(1.920 \mathrm{~g} \mathrm{ha}^{-1}\right)$; mesotrione + ametryn $\left(120 \mathrm{~g}+2.000 \mathrm{~g}^{-1} \mathrm{a}^{-1}\right)$; mesotrione + metribuzin $\left(120 \mathrm{~g}+1920 \mathrm{~g} \mathrm{ha}^{-1}\right)$ e testemunhas no mato e no limpo. Concluiu-se que os herbicidas isolados ou em mistura foram seletivos à cana-de-açúcar. Com relação à eficácia, observou-se que o herbicida mesotrione foi eficiente no controle de A. deflexus; ametryn, no controle de A. deflexus, B. pilosa e I. nil; metribuzin, no controle de A. deflexus, B. pilosa e S. glaziovii; mesotrione + ametryn, no controle de $B$. decumbens, $B$. plantaginea, $D$. horizontalis, $P$. maximum, A. deflexus, B. pilosa, I. nil e S. glaziovii; e mesotrione + metribuzin, no controle de B. plantaginea, D. horizontalis, P. maximum, A. deflexus, B. pilosa e S. glaziovii. Foi constatado elevado efeito sinergístico do mesotrione com os herbicidas testados, sendo o efeito mais pronunciado na mistura com o ametryn.
\end{abstract}

Palavras-chave: controle químico, plantas daninhas, mistura, Saccharum spp.

ABSTRACT - There are many herbicide application options for sugarcane pre-emergence but, currently, post-emergence control is the great challenge. The objective of this work was to evaluate the efficiency of the herbicide mesotrione in mixture with ametryn and metribuzin in the post-emergence control of ten weeds sown in sugarcane, variety RB86-7515. The weeds used in the experiment were: Brachiaria decumbens, Brachiaria plantaginea, Cenchrus echinatus, Digitaria horizontalis, Panicum maximum, Amaranthus deflexus, Bidens pilosa, Euphorbia heterophylla, Ipomoea nil and Sida glaziovii, sown in between the rows after crop emergence. The herbicides were applied on the 45th day after sugarcane planting when the monocotyledon weeds reached the third tiller phase and the dicotyledon weeds completed three to four leaves. The following treatments were carried out: mesotrione (120 g ha $\left.\mathrm{g}^{-1}\right)$; ametryn (2,000 $\mathrm{g} \mathrm{ha}$ ); metribuzin $\left(1,920 \mathrm{~g} \mathrm{ha}^{-1}\right)$; mesotrione + ametryn $\left(120 \mathrm{~g}+2,000 \mathrm{~g} \mathrm{ha}^{-1}\right)$; mesotrione + metribuzin $(120 \mathrm{~g}+$ $1,920 \mathrm{~g} \mathrm{ha}^{-1}$ ) and control with and without weeds. It was concluded that the herbicides, isolated or in mixture, were selective to sugarcane. Regarding control efficiency, it was observed that the herbicide mesotrione was efficient in the control of $\boldsymbol{A}$. deflexus; ametryn was efficient in the control of A. deflexus, B. pilosa and I. nil; metribuzin was efficient in the control of A. deflexus, B. pilosa and S. glaziovii; mesotrione + ametryn were efficient in the control of $\boldsymbol{B}$. decumbens, B. plantaginea, D. horizontalis, P. maximum, A. deflexus, B. pilosa, I. nil and

Recebido para publicação em 10.3.2009 e na forma revisada em 3.9.2010.

2 Professor, Dr., Faculdade de Engenharia de Ilha Solteira, Universidade Estadual Paulista "Julio de Mesquita Filho" FEIS/UNESP, Av. Brasil, 56, Centro, 15385-000 Ilha Solteira-SP, ${ }^{3}$ Pós-graduandos em Agronomia, UNESP; 4 Professor, Dr., Universidade Anhanguera-Uniderp, Campo Grande-MS.

Planta Daninha, Viçosa-MG, v. 28, n. 3, p. 585-590, 2010 
S. glaziovii, and mesotrione + metribuzin was efficient in the control of B. plantaginea, D. horizontalis, $\boldsymbol{P}$. maximum, A. deflexus, B. pilosa and S. glaziovii. A high synergistic effect of mesotrione was verified with the tested herbicides, with the mixture with ametryn being the most prominent.

Keywords: chemical control, weeds, mixture, Saccharum spp.

\section{INTRODUÇÃO}

O Brasil é o maior produtor de cana-deaçúcar do mundo. Na safra 2007, a área cultivada foi de 6,62 milhões de hectares, estando a maior concentração no Estado de São Paulo, com 3,45 milhões de hectares (CONAB, 2007). As condições edafoclimáticas do Brasil são favoráveis à produção agrícola e, em especial, à cana-de-açúcar, porém existe uma série de fatores que podem prejudicar a sua produção. Um dos pontos críticos no processo produtivo da cultura é a interferência imposta pelas plantas daninhas, ao competirem com a cultura por nutrientes, água e luz e dificultarem o corte e a colheita, diminuindo a qualidade do produto colhido e reduzindo a longevidade do canavial (Kuva et al., 2003).

Na cultura da cana-de-açúcar, a utilização de herbicidas em pós-emergência é limitada, devido às poucas opções de herbicidas seletivos. Como muitas vezes a aplicação em pós-emergência se faz necessária, têm-se adotado aplicações de herbicidas não seletivos em jato dirigido ou o emprego dos recomendados para a pré-emergência que possuem efeito em pósemergência inicial. Um dos herbicidas mais utilizados como pós-emergente seletivo para a cana-de-açúcar é o ametryn (Agrianual, 2005).

A cultura da cana-de-açúcar desenvolvese bem em diferentes situações de manejo (cana-planta; cana-soca sem palha; cana-soca com palha; cana-de-açúcar de terceiro e quarto cortes, etc.). Entretanto, essa grande variação nos fatores ecológicos proporciona nichos adequados para o desenvolvimento de um grande número de espécies daninhas. Dessa forma, os herbicidas precisam apresentar amplo espectro de controle para se adequar às exigências da cultura. Isso explica as várias opções de misturas formuladas de herbicidas (Rodrigues \& Almeida, 2005) existentes para a cana-de-açúcar. A mistura de herbicidas é uma alternativa, pois visa aumentar o espectro de controle das plantas daninhas e a eficácia dos herbicidas.

Existem muitas opções de herbicidas para aplicação em pré-emergência na canade-açúcar, mas para aplicação em pósemergência o número é limitado. O herbicida mesotrione é registrado para o controle em pós-emergência de plantas daninhas na cultura do milho (Rodrigues \& Almeida, 2005). Por apresentar seletividade também para as plantas de cana-de-açúcar, possui potencial para ser usado nessa cultura. Entretanto, como o espectro de ação do mesotrione é relativamente pequeno para ser aplicado isoladamente em cana-de-açúcar, tendo em vista o grande número de espécies daninhas que infestam a cultura, sugere-se o emprego de misturas que proporcionem efeitos sinergísticos no controle das plantas daninhas.

O objetivo deste trabalho foi avaliar a seletividade à cultura e a eficácia do herbicida mesotrione em mistura com ametryn e metribuzin no controle em pós-emergência de 10 espécies daninhas semeadas em cana-de-açúcar.

\section{MATERIAL E MÉTODOS}

O trabalho foi conduzido no período de março/2005 a fevereiro/2006 na área de plantio da Usina Pioneiros Bioenergia, município de Ilha Solteira, na região noroeste do Estado de São Paulo $\left(20^{\circ} 23^{\prime} 47^{\prime \prime} \mathrm{S}, 5^{\circ} 13^{\prime} 10^{\prime \prime} \mathrm{W}\right.$ e $340 \mathrm{~m}$ de altitude). O solo da área experimental é classificado como Latossolo Vermelho-Escuro, textura média, com $32 \%$ de argila, $60 \%$ de areia e $8 \%$ de silte. O cultivar de cana-deaçúcar utilizado foi RB 86-7515, plantado em 1/3/2005, de forma convencional, no espaçamento de $1,50 \mathrm{~m}$. Os tratos culturais realizados na área experimental foram os recomendados para a cultura, no que diz respeito às adubações e ao controle de pragas e doenças. 
As espécies daninhas selecionadas para o experimento - Brachiaria decumbens, Brachiaria plantaginea, Cenchrus echinatus, Digitaria horizontalis, Panicum maximum, Amaranthus deflexus, Bidens pilosa, Euphorbia heterophylla, Ipomoea nil e Sida glaziovii-foram semeadas nas entrelinhas após a emergência da cultura, numa proporção média de 100 sementes por metro. O delineamento experimental foi o de blocos ao acaso, com sete tratamentos e quatro repetições, com parcelas de $2 \times 5 \mathrm{~m}\left(10 \mathrm{~m}^{2}\right)$, para cada espécie daninha. Os herbicidas foram aplicados aos 45 dias após o plantio da cana-de-açúcar, com as poáceas na fase de terceiro perfilho; as dicotiledôneas, com três a quatro pares de folhas; e a cultura, com três a quatro folhas. Os tratamentos empregados foram: mesotrione (120 $\left.\mathrm{g} \mathrm{ha}^{-1}\right)$, ametryn (2.000 $\left.\mathrm{g} \mathrm{ha}^{-1}\right)$, metribuzin $\left(1.920 \mathrm{~g} \mathrm{ha}^{-1}\right)$, mesotrione + ametryn $(120 \mathrm{~g}$ $+2.000 \mathrm{~g} \mathrm{ha}^{-1}$, mesotrione + metribuzin $\left(120 \mathrm{~g}+1.920 \mathrm{~g} \mathrm{ha}^{-1}\right)$ e testemunhas no mato e no limpo. Os tratamentos químicos foram aplicados com o adjuvante Agral (0,1\% v/v). As aplicações dos herbicidas foram feitas com um pulverizador costal pressurizado $\left(\mathrm{CO}_{2}\right.$ a $45 \mathrm{lb}$ $\mathrm{pol}^{-2}$ ), provido de tanque com capacidade de dois litros e com barra equipada com quatro bicos do tipo leque, Teejet $110.03 \mathrm{XR}$, espaçados de $0,5 \mathrm{~m}$ e mantidos a $0,5 \mathrm{~m}$ do alvo (plantas daninhas). O volume de calda aplicado foi de $250 \mathrm{~L} \mathrm{ha}^{-1}$.

As avaliações de eficiência dos produtos foram realizadas aos 15, 30, 60, 90, $120 \mathrm{e}$ 150 dias após a aplicação (DAA) dos herbicidas, empregando-se a escala de notas em porcentagem, em que a ausência de injúria correspondeu ao valor zero e a morte das plantas, ao valor 100. Considerou-se como eficiente o controle igual ou superior a $80 \%$ aos 120 DAA, época do fechamento da cultura. As avaliações de seletividade foram realizadas quinzenalmente até o desaparecimento dos sintomas, comparando-se os tratamentos com a testemunha no limpo e estimando-se a porcentagem de injúria causada pelos herbicidas.

A análise de crescimento das plantas da cultura foi o parâmetro que auxiliou na interpretação dos resultados de seletividade e interferência das plantas daninhas. Foram avaliadas as caracteristicas do desenvolvimento da cultura, aos 10 meses após o plantio, analisando-se o número de colmos em $5 \mathrm{~m}$; a altura de plantas, medida do colo ao final do último gomo; e o diâmetro do segundo gomo, em 10 plantas seguidas, por parcela. A avaliação de produtividade foi feita aos 12 meses após o plantio, coletando e pesando os colmos de cana-de-açúcar da área central de cada parcela $\left(7,5 \mathrm{~m}^{2}\right)$.

Os dados foram submetidos à análise da variância, empregando-se o teste F; quando significativas, as médias dos tratamentos foram comparadas pelo teste de Tukey a 5\%. Os dados de eficácia dos herbicidas foram analisados individualmente para cada espécie daninha. Os demais parâmetros (seletividade, biometria e produtividade) foram analisados dentro do conjunto das 10 espécies daninhas por parcela.

\section{RESULTADOS E DISCUSSÃO}

Os dados de controle das plantas daninhas aos 120 DAA estão apresentados nas Tabelas 1 e 2. Observou-se que o herbicida mesotrione aplicado isoladamente foi eficiente no controle de $A$. deflexus, proporcionando controle total $(100,0 \%)$, e pouco eficiente para as demais espécies. Deve-se ressaltar, porém, que o mesotrione é recomendado para aplicação em pós-emergência inicial (Rodrigues \& Almeida, 2005) e que, nesta pesquisa, as plantas daninhas já haviam passado dessa fase na época da aplicação.

A eficácia do mesotrione em pós-emergência inicial tem sido observada em várias espécies daninhas. Foloni (2002) observou, para a cultura do milho, a eficácia do mesotrione no controle de $D$. horizontalis, Eleusine indica, B. plantaginea, Acanthospermum hispidum, Portulaca oleracea e Galinsoga parviflora.

O herbicida ametryn foi eficiente no controle de A. deflexus, B. pilosa e I. nil. Apesar de o ametryn ser considerado eficiente para várias espécies em pós-emergência inicial (Lorenzi et al., 2006), no presente trabalho, de maneira geral, o tratamento foi pouco eficiente em razão da fase em que se encontravam as plantas daninhas na época da aplicação do herbicida. O metribuzin foi eficiente também no controle de três espécies daninhas, proporcionando controle de $83,0 \%$ para 
Tabela 1 - Eficácia dos tratamentos no controle das plantas daninhas monocotiledôneas. Ilha Solteira-SP (2005/06)

\begin{tabular}{|c|c|c|c|c|c|c|}
\hline \multirow{2}{*}{ Tratamento } & \multirow{2}{*}{$\begin{array}{c}\text { Dose } \\
\left(\mathrm{g} \mathrm{ha}^{-1}\right)\end{array}$} & \multicolumn{5}{|c|}{ Porcentagem de controle aos 120DAA } \\
\hline & & B. decumbens & B. plantaginea & C. echinatus & D. horizontalis & P. maximum \\
\hline 1. testemunha no mato & -- & $0,0 \mathrm{e}^{\underline{1}}$ & $0,0 \mathrm{e}$ & $0,0 \mathrm{f}$ & $0,0 \mathrm{~d}$ & $0,0 \mathrm{~g}$ \\
\hline 2. testemunha no limpo & -- & $100,0 \mathrm{a}$ & $100,0 \mathrm{a}$ & $100,0 \mathrm{a}$ & $100,0 \mathrm{a}$ & $100,0 \mathrm{a}$ \\
\hline 3. mesotrione & 120 & $18,8 \mathrm{~d}$ & $16,3 \mathrm{~d}$ & $48,8 \mathrm{~d}$ & $43,8 \mathrm{c}$ & $10,0 \mathrm{f}$ \\
\hline 4. ametryn & 2.000 & $57,5 \mathrm{~b}$ & $78,8 \mathrm{~b}$ & $62,5 \mathrm{bc}$ & $37,5 \mathrm{c}$ & $41,3 \mathrm{e}$ \\
\hline 5. metribuzin & 1.920 & $41,8 \mathrm{c}$ & $50,0 \mathrm{c}$ & $29,3 \mathrm{e}$ & $61,3 \mathrm{~b}$ & $58,0 \mathrm{~d}$ \\
\hline 6. mesotrione+ametryn & $120+2.000$ & $100,0 \mathrm{a}$ & $100,0 \mathrm{a}$ & $72,5 \mathrm{~b}$ & $90,0 \mathrm{a}$ & $91,3 \mathrm{~b}$ \\
\hline 7. mesotrione + metribuzin & $120+1.920$ & $48,8 \mathrm{bc}$ & $81,3 \mathrm{~b}$ & $57,5 \mathrm{~cd}$ & $100,0 \mathrm{a}$ & $81,3 \mathrm{c}$ \\
\hline \multicolumn{2}{|l|}{ Média Geral } & 52,39 & 60,89 & 52,93 & 61,79 & 54,54 \\
\hline \multicolumn{2}{|c|}{ Teste $\mathrm{F}$ (tratamentos) } & $256^{* *}$ & $442 * *$ & $132 * *$ & $208^{* *}$ & $1.435 * *$ \\
\hline \multicolumn{2}{|c|}{ Coeficiente de Variação (\%) } & 9,01 & 6,25 & 10,47 & 8,42 & 3,81 \\
\hline \multicolumn{2}{|c|}{$\operatorname{DMS}(5 \%)$} & 11,02 & 8,89 & 12,94 & 12,15 & 4,85 \\
\hline
\end{tabular}

1/ Médias seguidas por letras iguais na coluna não diferem entre si pelo teste de Tukey a $5 \%$ de probabilidade.

Tabela 2 - Eficácia dos tratamentos no controle das plantas daninhas dicotiledôneas. Ilha Solteira-SP (2005/06)

\begin{tabular}{|c|c|c|c|c|c|c|}
\hline \multirow{2}{*}{ Tratamento } & \multirow{2}{*}{$\begin{array}{c}\text { Dose } \\
\left(\mathrm{L} \mathrm{ha}^{-1}\right)\end{array}$} & \multicolumn{5}{|c|}{ Porcentagem de controle aos 120 DAA } \\
\hline & & A.deflexus & B. pilosa & E.heterophylla & I.nil & S.glaziovii \\
\hline 1. testemunha no mato & -- & $0,0 \mathrm{c}^{\underline{1}}$ & $0,0 \mathrm{~d}$ & $0,0 \mathrm{f}$ & $0,0 \mathrm{e}$ & $0,0 \mathrm{f}$ \\
\hline 2. testemunha no limpo & -- & $100,0 \mathrm{a}$ & $100,0 \mathrm{a}$ & $100,0 \mathrm{a}$ & $100,0 \mathrm{a}$ & $100,0 \mathrm{a}$ \\
\hline 3. mesotrione & 120 & $100,0 \mathrm{a}$ & $5,0 \mathrm{c}$ & $21,3 \mathrm{e}$ & $66,3 \mathrm{~d}$ & $31,3 \mathrm{e}$ \\
\hline 4. ametryn & 2.000 & $100,0 \mathrm{a}$ & $100,0 \mathrm{a}$ & $61,3 \mathrm{bc}$ & $85,0 \mathrm{bc}$ & $48,8 \mathrm{~d}$ \\
\hline 5. metribuzin & 1.920 & $83,0 \mathrm{~b}$ & $82,8 \mathrm{~b}$ & $39,5 \mathrm{~d}$ & $63,0 \mathrm{~d}$ & $80,0 \mathrm{c}$ \\
\hline 6. mesotrione+ametryn & $120+2.000$ & $100,0 \mathrm{a}$ & $100,0 \mathrm{a}$ & $70,0 \mathrm{~b}$ & $92,5 \mathrm{ab}$ & $95,0 \mathrm{~b}$ \\
\hline 7. mesotrione + metribuzin & $120+1.920$ & $100,0 \mathrm{a}$ & $100,0 \mathrm{a}$ & $53,8 \mathrm{c}$ & $78,8 \mathrm{c}$ & $100,0 \mathrm{a}$ \\
\hline \multicolumn{2}{|l|}{ Média Geral } & 83,29 & 69,68 & 49,39 & 69,36 & 62,32 \\
\hline \multicolumn{2}{|c|}{ Teste F (tratamentos) } & $6.482 * *$ & $12.232^{* *}$ & $260^{* *}$ & $376^{* *}$ & $1.030^{* *}$ \\
\hline \multicolumn{2}{|c|}{ Coeficiente de Variação (\%) } & 1,11 & 1,20 & 8,24 & 4,96 & 4,01 \\
\hline \multicolumn{2}{|c|}{ DMS $(5 \%)$} & 2,16 & 1,96 & 9,50 & 8,03 & 5,84 \\
\hline
\end{tabular}

${ }^{1 /}$ Médias seguidas por letras iguais na coluna não diferem entre si pelo teste de Tukey a $5 \%$ de probabilidade.

A. deflexus, de $82,8 \%$ para B. pilosa e de $80,0 \%$ para S. glaziovii.

O tratamento mesotrione + ametryn foi eficiente no controle de Brachiaria decumbens, $B$. plantaginea, D. horizontalis, P. maximum, A. deflexus, B. pilosa, I. nil e S. glaziovii. Para as outras duas espécies, o tratamento foi pouco eficiente, proporcionando controle de $72,5 \%$ para Cenchrus echinatus e de $70,0 \%$ para Euphorbia heterophylla, aos 120 DAA.

Os resultados obtidos para o tratamento mesotrione + ametryn $\left(120 \mathrm{~g}+2.000 \mathrm{~g} \mathrm{ha}^{-1}\right)$ revelaram elevado efeito sinergístico entre os herbicidas. Observa-se pelos dados de controle que, para todas as espécies daninhas estudadas, o efeito da mistura foi maior do que a eficácia de cada um dos herbicidas aplicados isoladamente, exceto para Amaranthus deflexus, que foi totalmente controlada pelos próprios produtos isolados. O resultado alcançado pelo tratamento representa uma nova opção de manejo para a cultura da cana-de-açúcar, possibilitando o controle seletivo mais tardio, com a vantagem de se obter o fechamento da cultura no limpo com apenas uma aplicação. No trabalho de Fornarolli et al. (1997) são apresentados resultados de controle de plantas daninhas em pós-emergência tardia com o uso de MSMA + diuron em jato dirigido na cultura da cana-de-açúcar.

O tratamento mesotrione + metribuzin $\left(120 \mathrm{~g}+1.920 \mathrm{~g} \mathrm{ha}^{-1}\right)$ foi eficiente no controle de seis espécies daninhas, proporcionando controle total $(100,0 \%)$ para $D$. horizontalis, 
A. deflexus e $S$. glaziovii e controle de $81,3 \%$ para B. plantaginea e $P$. maximum. Para I. nil, $B$. decumbens, $C$. echinatus e $E$. heterophylla, a porcentagem de controle foi inferior a $80 \%$. Apesar de os resultados finais serem inferiores ao da mistura mesotrione + ametryn, observou-se também sinergismo entre esses herbicidas. Dessa forma, a opção por utilizar o ametryn ou o metribuzin em mistura com mesotrione depende das espécies daninhas presentes na área e do preço dos herbicidas.

Os dados médios de fitotoxicidade dos herbicidas estão apresentados na Tabela 3. Todos eles provocaram sintomas aceitáveis de toxicidade inicial às plantas de cana-de-açúcar. Aos 30 DAA observou-se redução gradativa dos sintomas e, aos 45 DAA, não foi mais observado nenhum sintoma em plantas da cultura. Os indices de fitotoxicidade observados no experimento demonstram que todos os tratamentos foram seletivos às plantas de cana-de-açúcar.

Os dados de crescimento e produtividade da cultura (Tabela 4) confirmam a seletividade dos tratamentos. Quanto aos dados de diâmetro de colmos, altura média de plantas, número de perfilhos e produtividade da cana-de-açúcar, observou-se que todos os tratamentos mostraram valores maiores do que os observados na testemunha no mato. Além disso, os tratamentos mais eficientes no manejo apresentaram maior produtividade, comprovando que a convivência com as plantas daninhas prejudica a cana-de-açúcar. Assim, podem-se

Tabela 3 - Seletividade dos tratamentos às plantas de cana-de-açúcar. Ilha Solteira-SP (2005/06)

\begin{tabular}{|c|c|c|c|c|}
\hline \multirow{2}{*}{ Tratamento } & \multirow{2}{*}{$\begin{array}{c}\text { Dose } \\
\left(\mathrm{L} \mathrm{ha}^{-1}\right)\end{array}$} & \multicolumn{3}{|c|}{$\%$ Fitotoxicidade } \\
\hline & & $15 \mathrm{DAA}$ & $30 \mathrm{DAA}$ & $45 \mathrm{DAA}$ \\
\hline 1. testemunha no mato & -- & $0,0 \mathrm{c}$ & $0,0 \mathrm{e}$ & 0,0 \\
\hline 2. testemunha no limpo & -- & $0,0 \mathrm{c}$ & $0,0 \mathrm{e}$ & 0,0 \\
\hline 3. mesotrione & 120 & $4,0 \mathrm{~b}$ & $1,8 \mathrm{~cd}$ & 0,0 \\
\hline 4. ametryn & 2.000 & $6,8 \mathrm{~b}$ & $4,0 \mathrm{bc}$ & 0,0 \\
\hline 5. metribuzin & 1.920 & $4,5 \mathrm{~b}$ & $2,0 \mathrm{a}$ & 0,0 \\
\hline 6. mesotrione + ametryn & $120+2.000$ & $14,8 \mathrm{a}$ & $7,8 \mathrm{ab}$ & 0,0 \\
\hline 7. mesotrione + metribuzin & $120+1.920$ & $13,0 \mathrm{a}$ & $5,0 \mathrm{ab}$ & 0,0 \\
\hline \multicolumn{2}{|l|}{ Média Geral } & 6,14 & 2,93 & - \\
\hline \multicolumn{2}{|l|}{ Teste F (tratamentos) } & $56,16^{* *}$ & $28,96^{* *}$ & - \\
\hline \multicolumn{2}{|l|}{ Coeficiente de Variação (\%) } & 25,35 & 35,93 & - \\
\hline \multicolumn{2}{|l|}{ DMS $(5 \%)$} & 3,64 & 2,46 & - \\
\hline
\end{tabular}

${ }^{1 /}$ Médias seguidas por letras iguais na coluna não diferem entre si pelo teste de Tukey a $5 \%$ de probabilidade.

Tabela 4 - Efeito dos tratamentos no crescimento e na produtividade da cana-de-açúcar. Ilha Solteira-SP (2005/06)

\begin{tabular}{|c|c|c|c|c|c|}
\hline \multirow[b]{2}{*}{ Tratamento } & \multirow[b]{2}{*}{$\begin{array}{c}\text { Dose } \\
\left(\mathrm{L} \mathrm{ha}^{-1}\right)\end{array}$} & \multicolumn{3}{|c|}{10 Meses Após o Plantio (MAP) } & \multirow{2}{*}{$\begin{array}{c}12 \text { Meses } \\
\text { Produtividade } \\
\left(\mathrm{t} \mathrm{ha}^{-1}\right)\end{array}$} \\
\hline & & $\begin{array}{l}\text { Diâmetro } \\
(\mathrm{mm})\end{array}$ & $\begin{array}{l}\text { Altura } \\
\text { (cm) }\end{array}$ & $\begin{array}{c}\mathrm{N}^{\mathrm{o}} \text { de } \\
\text { perfilhos } \mathrm{m}^{-1}\end{array}$ & \\
\hline 1. testemunha no mato & -- & $28,10 \mathrm{c}$ & $187,2 \mathrm{~d}$ & $9,3 \mathrm{c}$ & $91,45 \mathrm{e}$ \\
\hline 2. testemunha no limpo & -- & $30,20 \mathrm{ab}$ & $201,6 \mathrm{a}$ & $11,2 \mathrm{a}$ & $127,35 \mathrm{a}$ \\
\hline 3. mesotrione & 120 & $29,15 \mathrm{bc}$ & $187,3 \mathrm{~d}$ & $9,9 \mathrm{bc}$ & $101,10 \mathrm{de}$ \\
\hline 4. ametryn & 2.000 & $30,30 \mathrm{ab}$ & $192,9 \mathrm{bc}$ & $10,1 \mathrm{bc}$ & $110,70 \mathrm{bcd}$ \\
\hline 5. metribuzin & 1.920 & $30,05 \mathrm{ab}$ & $190,6 \mathrm{~cd}$ & $10,0 \mathrm{bc}$ & $106,90 \mathrm{~cd}$ \\
\hline 6. mesotrione + ametryn & $120+2.000$ & $30,42 \mathrm{a}$ & $198,2 \mathrm{a}$ & $10,9 \mathrm{ab}$ & $123,25 \mathrm{ab}$ \\
\hline 7. mesotrione + metribuzin & $120+1.920$ & $30,28 \mathrm{ab}$ & $197,6 \mathrm{ab}$ & $10,7 \mathrm{ab}$ & $120,05 \mathrm{abc}$ \\
\hline \multicolumn{2}{|c|}{ Média Geral } & 29,79 & 193,63 & 10,31 & 111,54 \\
\hline \multicolumn{2}{|c|}{ Teste F (tratamentos) } & $10,36 * *$ & $26,48 * *$ & $8,70^{* *}$ & $19,35 * *$ \\
\hline \multicolumn{2}{|c|}{ Coeficiente de Variação $(\%)$} & 1,79 & 1,14 & 4,33 & 5,25 \\
\hline \multicolumn{2}{|c|}{$\operatorname{DMS}(5 \%)$} & 1,24 & 5,13 & 1,04 & 13,66 \\
\hline
\end{tabular}

${ }^{1 /}$ Médias seguidas por letras iguais na coluna não diferem entre si pelo teste de Tukey a $5 \%$ de probabilidade. 
destacar como mais eficientes e mais produtivos os tratamentos: testemunha no limpo, mesotrione + ametryn e mesotrione + metribuzin.

Com relação aos efeitos das plantas daninhas na produtividade da cana-de-açúcar, pode-se afirmar que os prejuízos são resultantes do menor crescimento vegetativo da cultura, devido aos efeitos da interferência. A redução da produtividade da cana-de-açúcar devido à ocorrência de plantas daninhas também foi observada por Cruz \& Leiderman (1978), Clement et al. (1979), Victória Filho \& Camargo (1980) e Fornarolli et al. (1997).

\section{LITERATURA CITADA}

\author{
ANUÁRIO DA AGRICULTURA BRASILEIRA - \\ AGRIANUAL. Cana-de-açúcar. São Paulo: FNP, 2005. \\ p. 261-277.
}

CLEMENT, A. A. et al. Controle de plantas daninhas em cana-de-açúcar (Saccharum officinarum) com hexazinone e suas misturas com diuron, em pré-emergência.

Planta Daninha, v. 2, n. 2, p. 89-95, 1979.

\section{COMPANHIA NACIONAL DE ABASTECIMENTO -} CONAB. Acompanhamento da Safra Brasileira - Canade-Açúcar - Safra 2007/2008 - Primeiro Levantamento Maio/2007. Disponível em: <http://www.conab.gov.br/ conabweb/download/safra/1levan_cana07.pdf.>Acesso em:15 de jun. 2007.
CRUZ, L. S. P.; LEIDERMAN, L. Competição entre quatro herbicidas indicados para o controle de plantas daninhas em cana-de-açúcar (Saccharum spp.). Planta Daninha, v. 1, n. 2 , p. $43-48,1978$

FOLONI, L. L. Callisto (mesotrione) - um novo herbicida pós-emergente para a cultura do milho (Zea mays L.). In: CONGRESSO BRASILEIRO DA CIÊNCIA DAS PLANTAS DANINHAS, 23., 2002, Gramado. Resumos... Londrina: SBCPD, 2002. p. 308

FORNAROLLI, D. A. et al. Controle do Sorghum arundinaceum em pós-emergência inicial e tardia com o uso de MSMA+diuron na cultura da cana-de-açúcar (Saccharum spp.). In: CONGRESSO BRASILEIRO DA CIÊNCIA DAS PLANTAS DANINHAS, 21., 1997, Caxambu, MG. Resumos... Viçosa, MG: SBCPD, 1997. p. 259

KUVA, M. A. et al. Períodos de interferência das plantas daninhas na cultura da cana-de-açúcar. III - capim-braquiária (Brachiaria decumbens) e capim-colonião (Panicum maximum). Planta Daninha, v. 21, n. 1, p. 37-44, 2003

LORENZI, H. et al. Manual de identificação e controle de plantas daninhas - plantio direto e convencional. 6.ed. Nova Odessa: Plantarum, 2006. 339 p.

RODRIGUES, B. N.; ALMEIDA, F. S. Guia de herbicidas. 5.ed. Londrina: Edição dos Autores, 2005. 592 p.

VICTÓRIA FILHO, R.; CAMARGO, P. N. Efeito de herbicidas nos teores de macronutrientes e nas características tecnológicas da cana-de-açúcar (Saccharum spp.). I Misturas de herbicidas em pós-emergência. Planta Daninha, v. 3, n. 2, p. 96-107, 1980. 